\title{
LncRNA BLACAT1 is involved in chemoresistance of non-small cell lung cancer cells by regulating autophagy
}

\author{
FENG-XIANG HUANG ${ }^{1 *}$, HONG-JIE CHEN $^{1 *}$, FU-XIA ZHENG $^{2}$, ZENG-YAN GAO ${ }^{3}$, PAN-FENG SUN ${ }^{4}$, \\ QIANG PENG ${ }^{1}$, YING LIU ${ }^{1}$, XIANG DENG ${ }^{1}$, YA-HUI HUANG ${ }^{1}$, CHANG ZHAO ${ }^{1}$ and LI-JUN MIAO ${ }^{1}$ \\ ${ }^{1}$ Department of Respiratory and Critical Care Medicine, The First Affiliated Hospital of Zhengzhou University; \\ ${ }^{2}$ Department of Lung Disease Medicine, Traditional Chinese Medicine Hospital of Henan Province, \\ Zhengzhou, Henan 450052; ${ }^{3}$ Department of Respiratory Medicine, The Affiliated Hospital of \\ Weifang Medical University, Weifang, Shandong 261031; ${ }^{4}$ Department of Respiratory Medicine, \\ The Affiliated Huaihe Hospital of Henan University, Kaifeng, Henan 475000, P.R. China
}

Received July 6, 2018; Accepted October 1, 2018

DOI: $10.3892 /$ ijo.2018.4614

\begin{abstract}
The aim of the present study was to determine the effect of the long non-coding RNA (lncRNA) bladder cancer-associated transcript 1 (BLACAT1) in chemoresistance of non-small cell lung cancer (NSCLC) cells. Expression of lncRNA BLACAT1, microRNA (miR)-17, autophagy-related protein 7 (ATG7), multidrug-resistance protein 1 (MRP1), and the autophagy-associated proteins light chain 3 (LC3)-II/LC3-I and Beclin 1 were detected using the reverse transcription-quantitative polymerase chain reaction and western blot analysis. Cell viability was determined using an MTT assay. The interaction between BLACAT1 and miR-17 was determined using RNA immunoprecipitation and RNA pull-down assays. A cisplatin (DDP)-resistant NSCLC cell A549/DDP xenograft model in nude mice was established to investigate the effect of BLACAT1 on the chemoresistance of NSCLC cells. Compared with in DDP-sensitive NSCLC cells, expression of BLACAT1, ATG7, MRP1, LC3-II/LC3-I and Beclin 1 was significantly upregulated in DDP-resistant NSCLC cells, whereas miR-17 was downregulated in DDP-resistant NSCLC cells. Short interfering RNA against BLACAT1 decreased the viability of DDP-resistant NSCLC cells. In addition, BLACAT1 interacted with miR-17, and negatively regulated miR-17. BLACAT1 promoted ATG7 expression through miR-17, and facilitated autophagy
\end{abstract}

Correspondence to: Dr Li-Jun Miao, Department of Respiratory and Critical Care Medicine, The First Affiliated Hospital of Zhengzhou University, 1 Jianshe Road, Zhengzhou, Henan 450052, P.R. China

E-mail: miaolily@126.com

*Contributed equally

Key words: long non-coding RNA bladder cancer-associated transcript 1, microRNA-17, autophagy-related 7, chemoresistance, autophagy, non-small cell lung cancer and promoted chemoresistance of NSCLC cells through miR-17/ATG7. Finally, in vivo experiments indicated that inhibition of BLACAT1 ameliorated the chemoresistance of NSCLC. BLACAT1 was upregulated in DDP-resistant NSCLC cells, and promoted autophagy and chemoresistance of NSCLC cells through the miR-17/ATG7 signaling pathway.

\section{Introduction}

Non-small cell lung cancer (NSCLC) remains the leading cause of mortality from cancer, and surgery combined with chemotherapy has increased the long-term survival rate of patients with NSCLC (1). Chemotherapy is effective by triggering the death of tumor cells, but the occurrence of drug resistance can limit its efficacy (2). Therefore, seeking a therapeutic approach for overcoming drug resistance is vital for the treatment of NSCLC. Autophagy is a process of self-eating in cells, and can remove toxic substances in cancer cells and is involved in cellular degradation to maintain homeostasis (3). Studies have revealed that the ability of autophagy to maintain homeostasis in cells has led to chemoresistance in a number of types of cancer, including osteosarcoma, bladder cancer and gastric cancer (4-6). Therefore, autophagy may be a novel target for overcoming drug resistance.

Autophagy-related protein 7 (ATG7) is an E1-like enzyme and consists of the $\mathrm{N}$-terminal domain and the $\mathrm{C}$-terminal domain $(7,8)$. It can activate autophagy-essential ubiquitin-like proteins ATG8 and ATG12, thus serving crucial functions in autophagy $(8,9)$. It has been identified that ATG7-induced autophagy enhances the production of neural crest cells by modifying the cell cycle (10). In addition, studies have indicated that ATG7-dependent autophagy modulates the progression of a number of types of cancer (11-13). ATG7 may be an important molecular target in the study of chemoresistance of cancer through the involvement in different signaling pathways $(14,15)$. Sun et al (16) identified that the long non-coding RNA (lncRNA) X-inactive specific transcript knockdown decreased chemoresistance of NSCLC cells by inhibiting autophagy, and ATG7 overexpression enhanced the chemoresistance of A549/DDP cells, which suggested that 
ATG7 enhanced chemoresistance of NSCLC cells to cisplatin (DDP) by promoting autophagy.

MicroRNAs (miRNAs/miR) are a type of small non-coding RNAs that regulate gene expression at the post-transcriptional level by binding to the 3'-untranslated region in a variety of biological and pathological processes (17). It has been identified that miRNAs serve a vital function in regulating chemoresistance of NSCLC cells $(18,19)$. Zhao et al (20) identified that miR-17 was downregulated in A549/DDP cells compared with in A549 cells, and decreased miR-17 expression maintained cisplatin resistance in NSCLC. Comincini et al (21) identified that ATG7 was a potential target of miR-17, and miR-17 was able to negatively regulate ATG7, thus improving the sensitivity to temozolomide in human glioblastoma cells. Therefore, miR-17/ATG7 may be involved in the regulation of chemoresistance of NSCLC cells.

LncRNAs are a class of non-coding RNAs of $>200$ nucleotides. Recently, it has been revealed that several lncRNAs regulate chemoresistance of NSCLC cells through different pathways $(22,23)$. Ye et al (24) identified that, compared with adjacent normal tissues, the lncRNA bladder cancer-associated transcript 1 (BLACAT1) was upregulated in NSCLC tissues, and inhibition of BLACAT1 suppressed NSCLC cell proliferation, migration and invasion (24). Furthermore, BLACAT1 was able to promote oxaliplatin resistance of gastric cancer via the miR-361/ATP-binding cassette transporter B1 pathway (25). Therefore, we hypothesized that IncRNA BLACAT1 may serve a function in promoting the chemoresistance of NSCLC cells. The bioinformatics software LncBase (version 2) was used to predict the binding sites between BLACAT1 and miR-17. Therefore, we hypothesized that BLACAT1 promoted autophagy through miR-17/ATG7 to enhance the chemoresistance of NSCLC cells.

In the present study, it was identified that BLACAT1 was upregulated in cisplatin (DDP)-resistant NSCLC cells, and confirmed the interaction of lncRNA BLACAT1 and miR-17. In addition, IncRNA BLACAT1 facilitated autophagy through the miR-17/ATG7 signaling pathway, thus promoting chemoresistance of NSCLC cells.

\section{Materials and methods}

Cell culture and transfection. DDP-sensitive cell lines (A549 and H1299) and DDP-resistant cell lines (A549/DDP and H1299/DDP) were purchased from the Cell Bank of the Chinese Academy of Sciences (Shanghai, China). All cell lines were cultured in RPMI-1640 medium (Gibco; Thermo Fisher Scientific, Inc., Waltham, MA, USA) supplemented with $10 \%$ fetal bovine serum (Gibco; Thermo Fisher Scientific, Inc.), $2 \mu \mathrm{mol} / 1 \mathrm{~L}$-glutamine, $100 \mathrm{U} / \mathrm{ml}$ penicillin and $100 \mu \mathrm{g} / \mathrm{ml}$ streptomycin at $37^{\circ} \mathrm{C}$ under humidified conditions containing $5 \% \mathrm{CO}_{2}$. In order to maintain DDP resistance of A549/DDP and H1299/DDP cells, cells were cultured with $5 \mu \mathrm{g} / \mathrm{ml}$ DDP.

For BLACAT1 knockdown, short interfering RNA targeting BLACAT1 (si-BLACAT1, 5'-CCAGTGCATGGT CCTTGACTTT-3') was synthesized by Shanghai GeneChem Co., Ltd. (Shanghai, China). pcDNA-BLACAT1 was constructed by inserting BLACAT1 cDNA into pcDNA3.1 (Invitrogen; Thermo Fisher Scientific, Inc.). miR-17 mimic
(5'-CAAAGUGCUUACAGUGCAGGUAG-3'), miR-17 inhibitor (5'-GUUUCACGAAUGUCACGUCCAUC-3') and scrambled miRNA control were synthesized by Shanghai GeneChem Co., Ltd. Cells were seeded at $6 \times 10^{3}$ cells/well in a 6 -well plate and cultured until reaching $50 \%$ confluence, prior to transfection with 70 nmol pcDNA-BLACAT1, si-BLACAT1, miR-17 mimic, miR-17 inhibitor or negative control vectors using Lipofectamine ${ }^{\circledR} 2000$ transfection reagent (Invitrogen; Thermo Fisher Scientific, Inc.), according to the manufacturer's protocol. Following transfection, the autophagy inhibitor 3-methyladenine (3-MA; $1 \mathrm{mM}$ ) was used to treat A549 cells for $2 \mathrm{~h}$. The autophagy agonist rapamycin $(100 \mathrm{nM})$ was used to treat A549/DDP cells for $4 \mathrm{~h}$.

Reverse transcription-quantitative polymerase chain reaction $(R T-q P C R)$. Total RNA was extracted from A549, H1299, A549/DDP and H1299/DDP cells using TRIzol ${ }^{\circledR}$ reagent (Invitrogen; Thermo Fisher Scientific, Inc.). A High Capacity cDNA Reverse Transcription kit (Applied Biosystems; Thermo Fisher Scientific, Inc.) was used for cDNA synthesis, according to the manufacturer's protocol. A SuperScript ${ }^{\mathrm{TM}}$ III Platinum ${ }^{\mathrm{TM}}$ SYBR $^{\text {TM }}$ Green One-Step qPCR kit (Invitrogen; Thermo Fisher Scientific, Inc.) was used, according to the manufacturer's protocol, to determine BLACAT1 and miR-17 expression. RT-qPCR was conducted for 35 cycles of a denaturing phase at $94^{\circ} \mathrm{C}$ for $1 \mathrm{~min}$, primer annealing at $60^{\circ} \mathrm{C}$ for $1.5 \mathrm{~min}$, and an extension at $72^{\circ} \mathrm{C}$ for $2 \mathrm{~min}$ in a QuantStudio 3 Real-Time PCR System (Applied Biosystems; Thermo Fisher Scientific, Inc.). Primers were provided as follows: BLACAT1, 5'-GAC AAAGCACAAGCGAAACAAG-3' (forward) and 5'-GGA CATCTGATAGCCTGGTGAC-3' (reverse); GAPDH, 5'-TGC ACCACCAACTGCTTAGC-3' (forward) and 5'-GGCATG GACTGTGGTCATGAG-3' (reverse); miR-17, 5'-CAGTAA AGGTAAGGAGAGCTCAATCTG-3' (forward) and 5'-CAT ACAACCACTAAGCTAAAGAATAATCTGA-3' (reverse); U6, 5'-CTCGCTTCGGCAGCACA-3' (forward) and 5'-AAC GCTTCACGAATTTGCGT-3' (reverse). The relative expression of BLACAT1 and miR-17 was analyzed using the $2^{-\Delta \Delta \mathrm{Cq}}$ method (26).

Xenograft model in nude mice. Male nude mice (BALB/c $\mathrm{nu} / \mathrm{nu}$; 5-week-old; 16-20 g) were purchased from the laboratory animal center of Zhengzhou University (Zhengzhou, China), and were housed in 12-h light/12-h dark sterile conditions $\left(26-28^{\circ} \mathrm{C}\right.$, relative humidity $\left.40-60 \%\right)$ with no limitation to water and food. Lenti-si-control or lenti-si-BLACAT1 was synthesized by Shanghai GeneChem Co., Ltd. and transfected into A549/DDP cells. A $100 \mu 1$ volume of PBS containing $1 \times 10^{7}$ A549/DDP cells was subcutaneously injected into the nude mice (7 mice in each group). Tumor growth was determined using a caliper every 7 days. Mice were sacrificed at 28 days after inoculation, and tumor tissues were collected. The animal experiment was approved by the Ethics Committee of The First Affiliated Hospital of Zhengzhou University.

Western blotting. Cells and tumors were collected and lysed in radioimmunoprecipitation assay buffer (Beyotime Institute of Biotechnology, Haimen, China). The concentration of proteins was determined using a Bicinchoninic Acid Protein assay kit (Beyotime Institute of Biotechnology). Equal amounts (25 g) 
of protein of each sample were separated by SDS-PAGE (12\% gel) and transferred onto a polyvinylidene difluoride membrane (Invitrogen; Thermo Fisher Scientific, Inc.). The membrane was incubated with primary antibodies against ATG7 (1:10,000; cat. no. ab133528; Abcam, Cambridge, MA, USA), multidrug-resistance protein 1 (MRP1; 1:50; cat. no. ab32574; Abcam), light chain 3 (LC3; 1:3,000; cat. no. ab51520; Abcam), Beclin 1 (1:2,000; cat. no. ab207612; Abcam) and $\beta$-actin (1:1,000; cat. no. ab8266; Abcam), and horseradish peroxidase-conjugated secondary antibody [goat anti-rabbit immunoglobulin G (IgG); 1:2,000; cat. no. ab97051; Abcam]. Blots were visualized using a ChemiDoc ${ }^{\mathrm{TM}} \mathrm{MP}$ Imaging system (Bio-Rad Laboratories, Inc., Hercules, CA, USA).

MTT assay. A549 or H1299 cells $\left(6 \times 10^{4}\right.$ cells $\left./ \mathrm{ml}\right)$ were seeded on a 96-well plate and incubated with various concentrations of DDP $(0,1,2,4$ and $8 \mu \mathrm{g} / \mathrm{ml})$. A549/DDP or H1299/DDP cells $\left(6 \times 10^{4}\right.$ cells $\left./ \mathrm{ml}\right)$ were incubated with various concentrations of DDP $(0,4,8,16$ and $32 \mu \mathrm{g} / \mathrm{ml})$. Following incubation at $37^{\circ} \mathrm{C}$ for $24 \mathrm{~h}$, MTT solution $(20 \mu \mathrm{l}$, $5 \mathrm{mg} / \mathrm{ml}$ ) was added to each well in the dark prior to further incubation at $37^{\circ} \mathrm{C}$ for $4 \mathrm{~h}$. Cells were lysed using dimethylsulfoxide (150 $\mu \mathrm{l} /$ well), and the reduction of MTT was quantified by determining the absorbance at $540 \mathrm{~nm}$ against a reference wavelength of $630 \mathrm{~nm}$ using a plate reader (Bio-Rad Laboratories, Inc.).

RNA immunoprecipitation (RIP). The bioinformatics software LncBase (version 2; diana.imis.athena-innovation. gr/DianaTools/index.php) was used to predict the binding sites between BLACAT1 and miR-17. A RIP assay was performed using a Magna RIP ${ }^{\text {TM }}$ RNA-Binding Protein Immunoprecipitation kit (EMD Millipore, Billerica, MA, USA). A549 cell lysate was prepared from $1.5 \times 10^{7}$ cells using $100 \mu 1$ RIP lysis buffer supplemented with $0.25 \mu 1$ RNase inhibitor and $0.5 \mu 1$ protease inhibitor. The cell lysate was centrifuged at $513 \mathrm{xg}$ for $15 \mathrm{~min}$ at $4^{\circ} \mathrm{C}$, and the supernatant was incubated with RIP buffer containing Protein A/G-Sepharose beads conjugated with anti-Argonaute 2 (AGO2) antibody (1:5,000; cat. no. 03-110; EMD Millipore) or negative control IgG (1:5,000; cat. no. 12-371; EMD Millipore). RNA-bound protein complex was obtained, and RT-qPCR was used to detect lncRNA BLACAT1 and miR-17 in the precipitates, as aforementioned.

RNA pull-down. The biotin-labeled lncRNA BLACAT1 was transcribed with the Biotin RNA Labeling mixture (Roche Diagnostics, Basel, Switzerland) and T7 RNA polymerase (Roche Diagnostics). A549 cell extract was prepared from $1.5 \times 10^{7}$ cells in RIP buffer, then mixed with biotin-labeled IncRNA BLACAT1 at $4^{\circ} \mathrm{C}$ for $1 \mathrm{~h}$. Beads were added prior to incubation at room temperature for $1 \mathrm{~h}$. Western blotting was used to detect AGO2 in the BLACAT1 pull-down complex, and RT-qPCR was used to detect miR-17 in the precipitates, as aforementioned.

Statistical analysis. All experiments were performed in triplicate. All data are expressed as the mean \pm standard deviation and analyzed using SPSS software (version 17.0;
SPSS, Inc., Chicago, IL, USA). The differences between groups were assessed by Student's t-test or one-way analysis of variance. $\mathrm{P}<0.05$ was considered to indicate a statistically significant difference.

\section{Results}

LncRNA BLACAT1 is upregulated in DDP-resistant NSCLC cells. To determine the expression of BLACAT1, miR-17 and ATG7 in NSCLC cells, RT-qPCR and western blot analysis were performed. As presented in Fig. 1A, lncRNA BLACAT1 was upregulated and miR-17 was downregulated in DDP-resistant cell lines (A549/DDP and H1299/DDP) compared with in the DDP-sensitive cell lines (A549 and H1299). Protein levels of ATG7 and MRP1 were upregulated in A549/DDP and H1299/DDP cell lines compared with in A549 and H1299 cell lines (Fig. 1B). The levels of the autophagy-associated proteins LC3-II/LC3-I and Beclin 1 were investigated further, and it was identified that the LC3-II/LC3-I ratio and Beclin 1 level were increased in A549/DDP and H1299/DDP cell lines (Fig. 1C).

IncRNA BLACAT1 is involved in the viability of DDP-resistant NSCLC cells. To investigate the function of BLACAT1 in the viability of NSCLC cells, pcDNA-BLACAT1 and si-BLACAT1 were used for BLACAT1 overexpression and BLACAT1 inhibition, respectively. A549 and H1299 cells were transfected with pcDNA or pcDNA-BLACAT1, and treated with DDP $(0$, 1, 2, 4 and $8 \mathrm{~g} / \mathrm{ml}$ ) for $24 \mathrm{~h}$. A549/DDP and H1299/DDP cells were transfected with si-control or si-BLACAT1 and treated with $\operatorname{DDP}(0,4,8,16$ and $32 \mu \mathrm{g} / \mathrm{ml})$ for $24 \mathrm{~h}$. It was observed that cell viability of A549 and H1299 cells was significantly increased in the pcDNA-BLACAT1 group compared with in the pcDNA group (Fig. 2A). The viability of A549/DDP and H1299/DDP cells was significantly decreased in the si-BLACAT1 group compared with in the si-control group (Fig. 2B).

Interaction between IncRNA BLACAT1 and miR-17. According to the bioinformatic software LncBase, there were binding sites between BLACAT1 and miR-17 (Fig. 3A). On the basis of the opposing expression of BLACAT1 and miR-17 in NSCLC cells in Fig. 1, we hypothesized that BLACAT1 may directly inhibit miR-17. The RIP assay identified that BLACAT1 and miR-17 were relatively enriched in AGO2 compared with IgG (Fig. 3B). The RNA pull-down detected AGO in the BLACAT1 pull-down complex (Fig. 3C), and miR-17 was identified to be accumulated in the BLACAT1 pull-down complex, compared with only a slight increase in NC (Fig. 3D). These results indicated that BLACAT1 interacted with miR-17, and could negatively regulate miR-17.

BLACAT1 promotes ATG7 expression through miR-17. A previous study identified that ATG7 was a potential target of miR-17, and miR-17 could negatively regulate ATG7 in human glioblastoma cells (21). On the basis of the consistent expression of BLACAT1 and ATG7, we hypothesized that BLACAT1 could positively regulate ATG7 expression through miR-17. As presented in Fig. 4A. miR-17 expression in A549 cells was significantly decreased following BLACAT1 overexpression, 

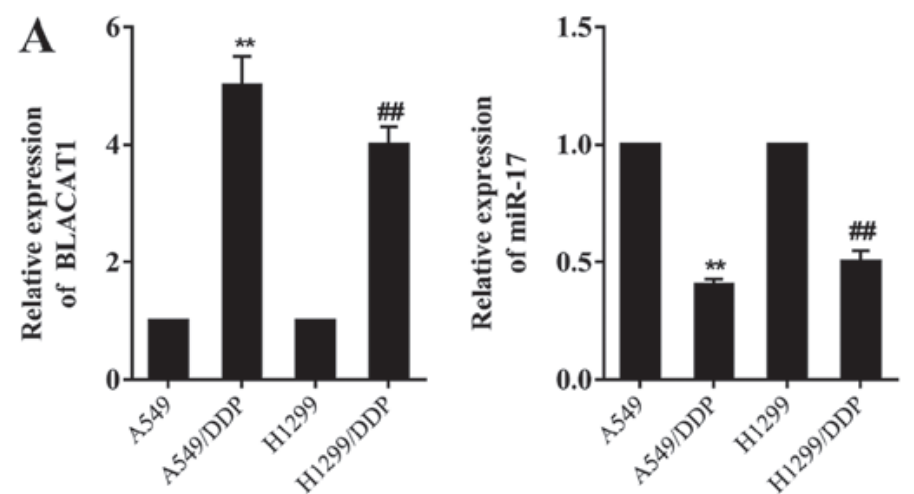

B

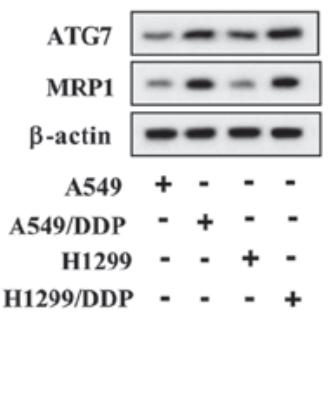

C

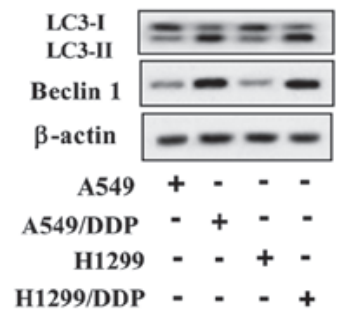

Figure 1. LncRNA BLACAT1 is upregulated in DDP-resistant NSCLC cells. (A) Compared with the DDP-sensitive cell lines A549 and H1299, lncRNA BLACAT1 was upregulated in the DDP-resistant cell lines A549/DDP and H1299/DDP, and miR-17 was downregulated in the DDP-resistant cell lines A549/DDP and H1299/DDP. (B) Protein levels of ATG7 and MRP1 were upregulated in the DDP-resistant cell lines A549/DDP and H1299/DDP. (C) The autophagy-associated protein LC3-II/LC3-I ratio was increased in the DDP-resistant cell lines A549/DDP and H1299/DDP, and the Beclin 1 level was also increased. " $\mathrm{P}<0.01$ vs. A549; "\#P $\mathrm{P} 0.01$ vs. H1299. BLACAT1, bladder cancer-associated transcript 1; lncRNA, long non-coding RNA; DDP, cisplatin; miR, microRNA; ATG7, autophagy-related protein 7; MRP1, multidrug-resistance protein 1; LC3, light chain 3.
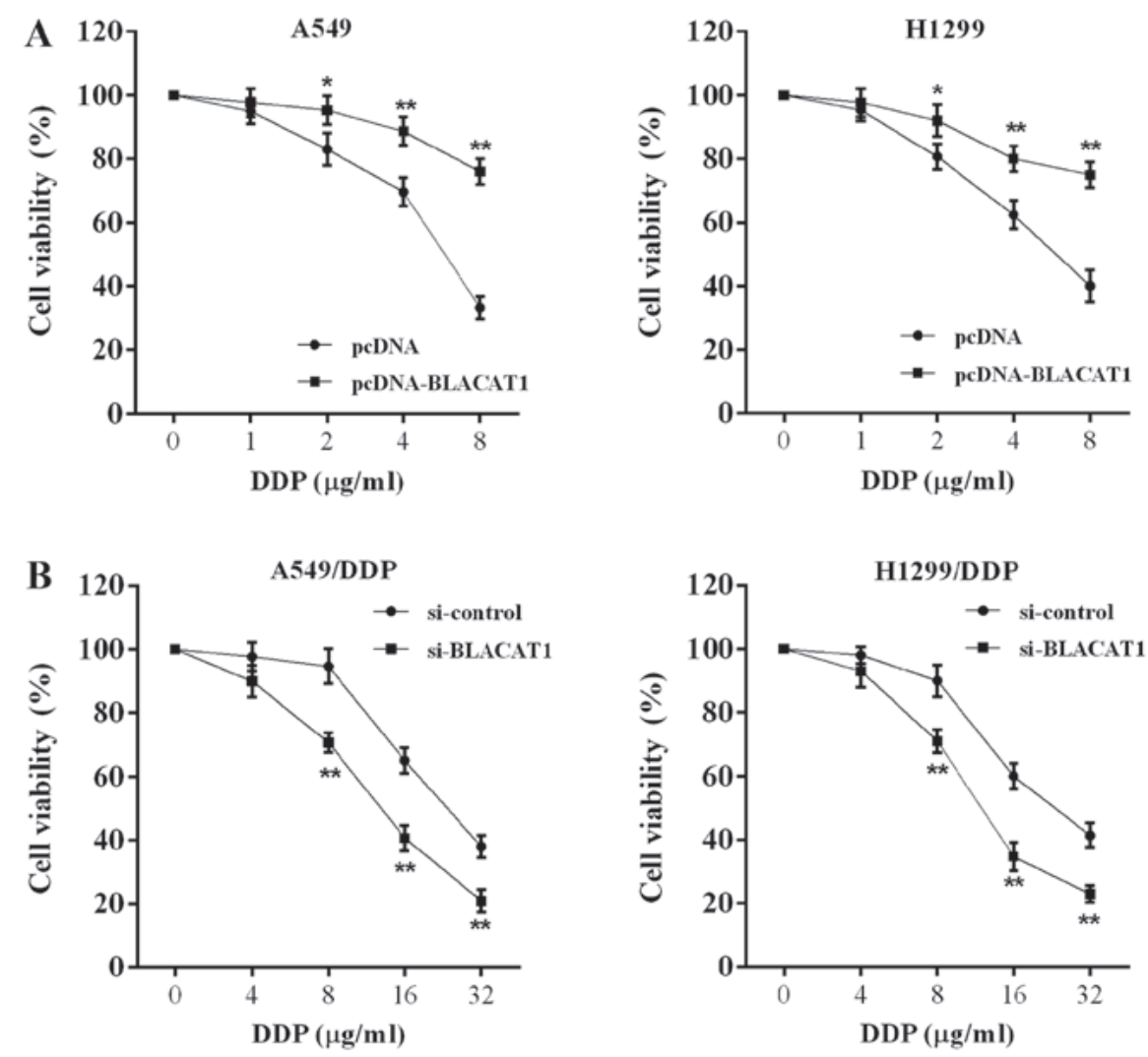

Figure 2. LncRNA BLACAT1 is involved in the viability of DDP-resistant NSCLC cells. A549 and H1299 cells were transfected with pcDNA or pcDNA-BLACAT1, and treated with DDP $(0,1,2,4$ and $8 \mu \mathrm{g} / \mathrm{ml})$ for $24 \mathrm{~h}$. A549/DDP and H1299/DDP cells were transfected with si-control or si-BLACAT1 and treated with DDP $(0,4,8,16$ and $32 \mu \mathrm{g} / \mathrm{ml})$ for $24 \mathrm{~h}$. (A) Viability of A549 and H1299 cells with lncRNA BLACAT1 overexpression was significantly increased. (B) Viability of A549/DDP and H1299/DDP cells with BLACAT1 inhibition was significantly decreased. "P $<0.05$, ${ }^{* * *} \mathrm{P}<0.01$ vs. pcDNA or si-control. BLACAT1, bladder cancer-associated transcript 1; lncRNA, long non-coding RNA; DDP, cisplatin; si, short interfering RNA.

and miR-17 mimic eliminated the inhibitory effect of BLACAT1 overexpression. The ATG7 protein level in A549 cells was upregulated following BLACAT1 overexpression, and miR-17 mimic eliminated the promotional effect of BLACAT1 overexpression (Fig. 4B). Following si-BLACAT1 transfection, miR-17 expression in A549/DDP cells was significantly increased, and miR-17 inhibitor eliminated the promotional effect of si-BLACAT1 (Fig. 4C). The ATG7 protein level in A549/DDP cells was downregulated following si-BLACAT1, and miR-17 inhibitor eliminated the inhibitory effect of si-BLACAT1 (Fig. 4D).

BLACAT1 facilitates autophagy through miR-17. To investigate whether BLACAT1 activated autophagy through miR-17, the LC3-I/LC3-II ratio and Beclin 1 expression were determined. It was identified that treatment with autophagy inhibitor 
A

BLACAT1

miR-17

chr1:205434958-205434975

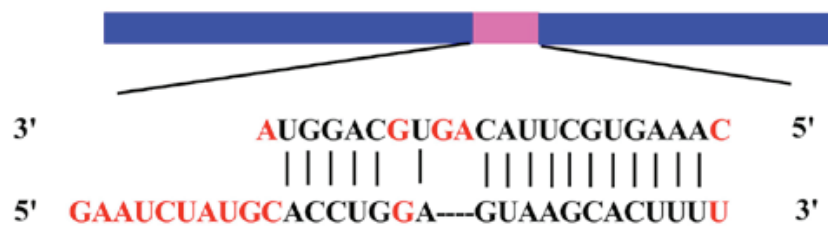

B
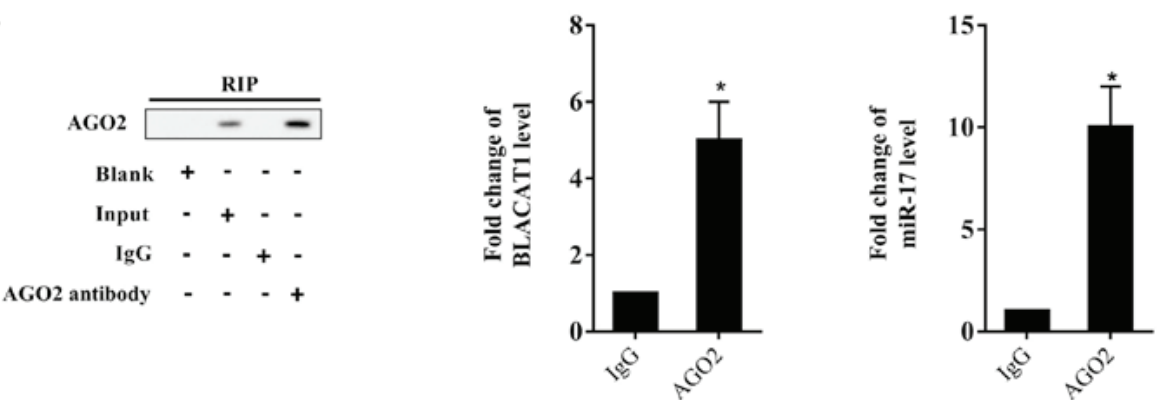

C
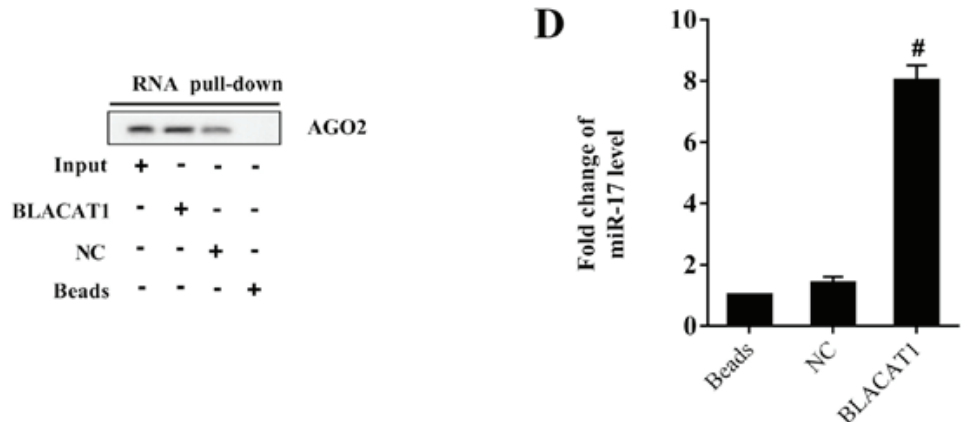

AGO2

Figure 3. Interaction between lncRNA BLACAT1 and miR-17. (A) Bioinformatic software predicted the binding sites between BLACAT1 and miR-17. (B) Anti-AGO2 antibody was used for the RIP assay. Compared with IgG, BLACAT1 and miR-17 were relatively enriched in AGO2. (C) RNA pull-down identified AGO in the BLACAT1 pull-down complex. (D) miR-17 was relatively enriched in the BLACAT1 pull-down complex, and slightly increased in NC "P<0.05 vs. IgG; " P <0.05 vs. NC. BLACAT1, bladder cancer-associated transcript 1; lncRNA, long non-coding RNA; miR, microRNA; AGO2, Argonaute 2; IgG, immunoglobulin G; NC, negative control; RIP, RNA immunoprecipitation.

A

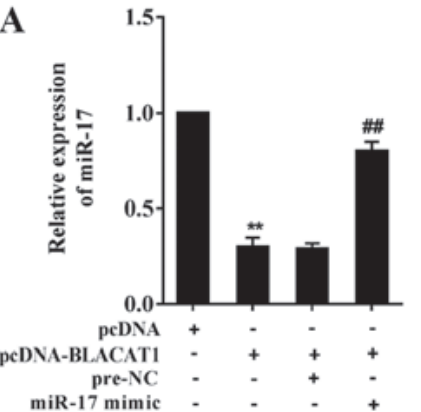

B

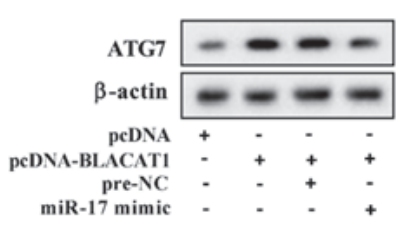

C

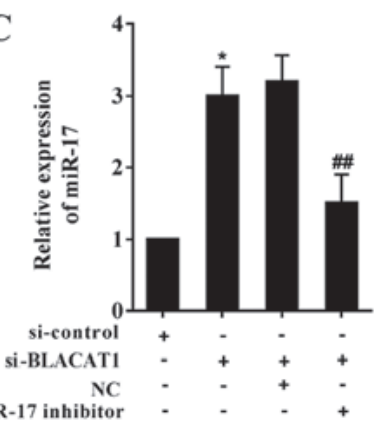

D

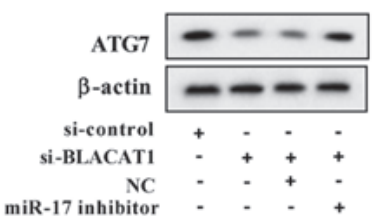

Figure 4. BLACAT1 promotes ATG7 expression through miR-17. A549 cells were divided into pcDNA, pcDNA-BLACAT1, pcDNA-BLACAT1+pre-NC and pcDNA-BLACAT1+miR-17 mimic groups. (A) miR-17 expression in A549 cells was significantly decreased following BLACAT1 overexpression, and miR-17 mimic eliminated the inhibitory effect of BLACAT1 overexpression. ${ }^{* *} \mathrm{P}<0.01 \mathrm{vs}$. pcDNA; ${ }^{\# /} \mathrm{P}<0.01 \mathrm{vs.} \mathrm{pcDNA-BLACAT1+pre-NC.} \mathrm{(B)} \mathrm{ATG7} \mathrm{protein} \mathrm{level} \mathrm{in}$ A549 cells was upregulated following BLACAT1 overexpression, and miR-17 mimic eliminated the promotional effect of BLACAT1 overexpression. A549/ DP cells were divided into si-control, si-BLACAT1, si-BLACAT1+NC and si-BLACAT1+miR-17 inhibitor groups. (C) miR-17 expression in A549/ DP cells was significantly increased following si-BLACAT1 treatment, and miR-17 inhibitor eliminated the promotional effect of si-BLACAT1. "P $<0.05$ vs. si-control; ${ }^{\# \#} \mathrm{P}<0.01$ vs. si-BLACAT1+NC. (D) ATG7 protein level in A549/DDP cells was downregulated following si-BLACAT1 treatment, and miR-17 inhibitor eliminated the inhibitory effect of si-BLACAT1. BLACAT1, bladder cancer-associated transcript 1; ATG7, autophagy-related protein 7; miR, microRNA; NC, negative control; si, short interfering RNA.

3-methyladenine (3-MA) suppressed autophagy of A549 cells. BLACAT1 overexpression promoted the conversion of LC3-I into LC3-II, and facilitated Beclin 1 expression in A549 cells, whereas miR-17 mimic eliminated the promotional effect of BLACAT1 overexpression (Fig. 5A). Following treatment with the autophagy agonist rapamycin, autophagy of A549/DDP cells was promoted. si-BLACAT1 inhibited the conversion of LC3-I into LC3-II, and suppressed Beclin 1 expression in A549/DDP cells, whereas miR-17 inhibitor eliminated the inhibitory effect of si-BLACAT1 (Fig. 5B). These results 

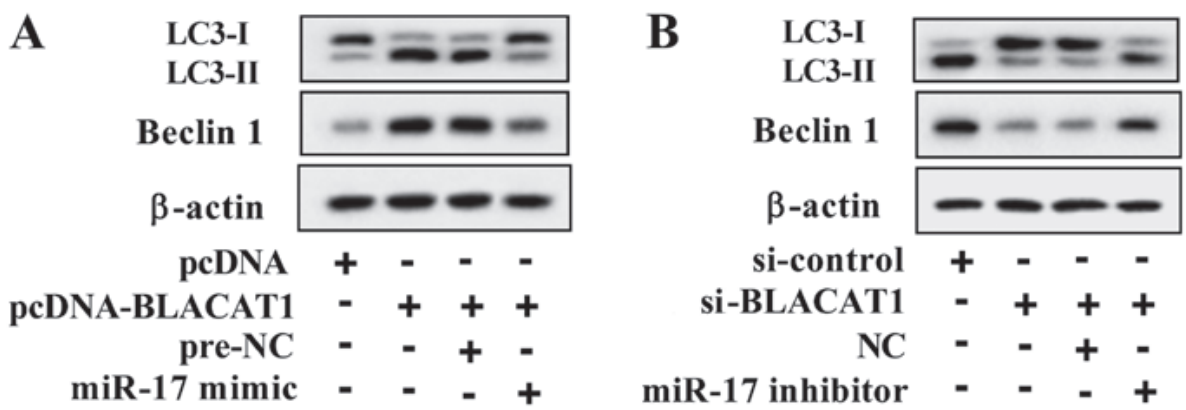

Figure 5. BLACAT1 facilitates autophagy through miR-17. (A) A549 cells were transfected with pcDNA, pcDNA-BLACAT1, pcDNA-BLACAT1+pre-NC and pcDNA-BLACAT1+miR-17 mimic groups. The autophagy inhibitor 3-methyladenine (1 mM) was used to treat A549 cells for $2 \mathrm{~h}$. BLACAT1 overexpression promoted the conversion of LC3-I into LC3-II, and facilitated Beclin 1 expression, whereas miR-17 mimic eliminated the promotional effect of BLACAT1 overexpression. (B) A549/DDP cells were transfected with si-control, si-BLACAT1, si-BLACAT1+NC and si-BLACAT1+miR-17 inhibitor. The autophagy agonist rapamycin (100 nM) was used to treat A549/DDP cells for $4 \mathrm{~h}$. si-BLACAT1 inhibited the conversion of LC3-I into LC3-II, and suppressed Beclin 1 expression, whereas miR-17 inhibitor eliminated the inhibitory effect of si-BLACAT1. BLACAT1, bladder cancer-associated transcript 1; miR, microRNA; LC3, light chain 3; si, short interfering RNA; NC, negative control; DDP, cisplatin.
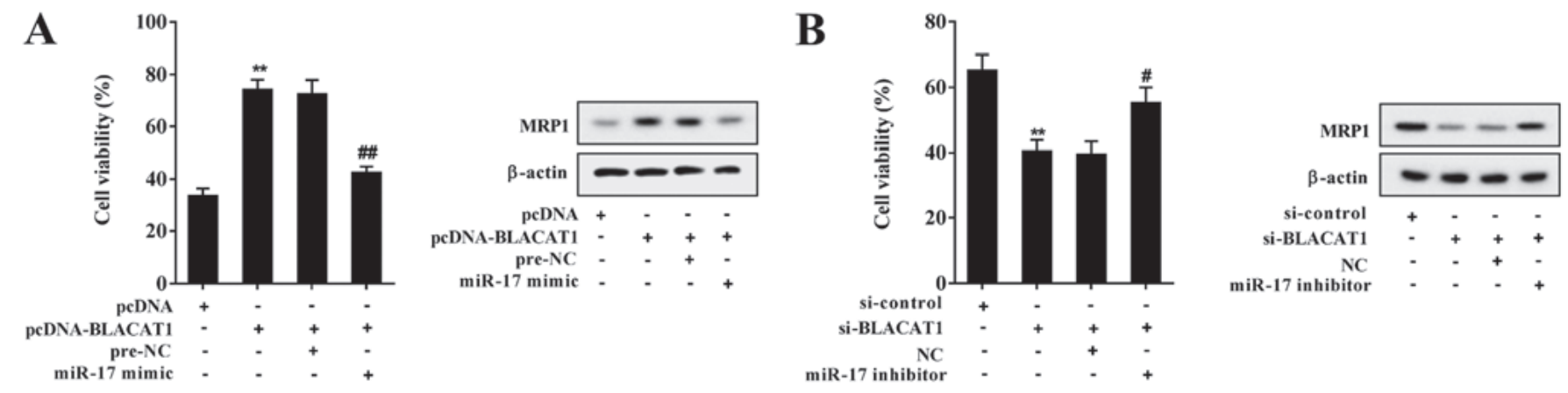

Figure 6. BLACAT1 promotes chemoresistance of non-small cell lung cancer cells through miR-17. (A) A549 cells were transfected with pcDNA, pcDNA-BLACAT1, pcDNA-BLACAT1+pre-NC and pcDNA-BLACAT1+miR-17 mimic. A549 cells were stimulated with $8 \mu \mathrm{g} / \mathrm{ml}$ DDP. BLACAT1 overexpression promoted chemoresistance of A549 cells and increased MRP1 expression, and miR-17 mimic eliminated the promotional effect of BLACAT1 overexpression. ${ }^{* *} \mathrm{P}<0.01$ vs. pcDNA; ${ }^{\# /} \mathrm{P}<0.01$ vs. pcDNA-BLACAT1+pre-NC. (B) A549/DDP cells were transfected with si-control, si-BLACAT1, si-BLACAT1+NC and si-BLACAT1+miR-17 inhibitor. A549/DDP cells were stimulated with $16 \mu \mathrm{g} / \mathrm{ml}$ DDP. si-BLACAT1 inhibited chemoresistance of A549/DDP cells and decreased MRP1 expression, and miR-17 inhibitor eliminated the inhibitory effect of si-BLACAT1. ${ }^{* *} \mathrm{P}<0.01 \mathrm{vs}$. si-control; ${ }^{*} \mathrm{P}<0.05$ vs. si-BLACAT1+NC. BLACAT1, bladder cancer-associated transcript 1; NC, negative control; DDP, cisplatin; MRP1, multidrug-resistant protein 1; miR, microRNA; si, short interfering RNA.

indicated that BLACAT1 upregulated autophagy-associated proteins to activate autophagy through inhibiting miR-17.

BLACAT1 promotes chemoresistance of NSCLC cells through $m i R-17$. The function of BLACAT1 in the chemoresistance of NSCLC cells was investigated further. Following stimulation with DDP, BLACAT1 overexpression promoted chemoresistance of A549 cells and increased MRP1 expression in A549 cells, and miR-17 mimic eliminated the promotional effect of BLACAT1 overexpression (Fig. 6A). Following stimulation with DDP, si-BLACAT1 inhibited chemoresistance of A549/DDP cells and decreased MRP1 expression, and miR-17 inhibitor eliminated the inhibitory effect of si-BLACAT1 (Fig. 6B).

BLACAT1 inhibition ameliorates chemoresistance of NSCLC in vivo. A549/DDP cells were transfected with lenti-si-control or lenti-si-BLACAT1, and the effect of BLACAT1 on chemoresistance in NSCLC in vivo was observed. Tumor volumes in mice of the lenti-si-BLACAT1 group were lower compared with those in the lenti-si-control group (Fig. 7A). As presented in Fig. 7B, BLACAT1 inhibition suppressed BLACAT1 and ATG7 expression, and promoted miR-17 expression of tumor tissues in the xenograft mouse model. Furthermore, BLACAT1 inhibition suppressed conversion of LC3-I into LC3-II, and inhibited Beclin 1 and MRP1 expression (Fig. 7C), which suggested that BLACAT1 inhibition ameliorated autophagy and chemoresistance in NSCLC.

\section{Discussion}

In the present study, it was identified that lncRNA BLACAT1, ATG7, MRP1 LC3-II/LC3-I and Beclin 1 were significantly upregulated in DDP-resistant NSCLC cells, whereas miR-17 was downregulated in DDP-resistant NSCLC cells. Inhibition of BLACAT1 significantly decreased the viability of A549/DDP and H1299/DDP cells following treatment with DDP. Furthermore, it was identified that BLACAT1 interacted with miR-17, and BLACAT1 negatively regulated miR-17. Inhibition of BLACAT1 decreased the LC3-II/LC3-I ratio and Beclin 1 expression, and decreased the viability of A549/DDP cells. In vivo experiments indicated that inhibition of BLACAT1 suppressed autophagy in NSCLC.

LncRNAs exert an important function in a variety of cancer types, and previous studies have identified 

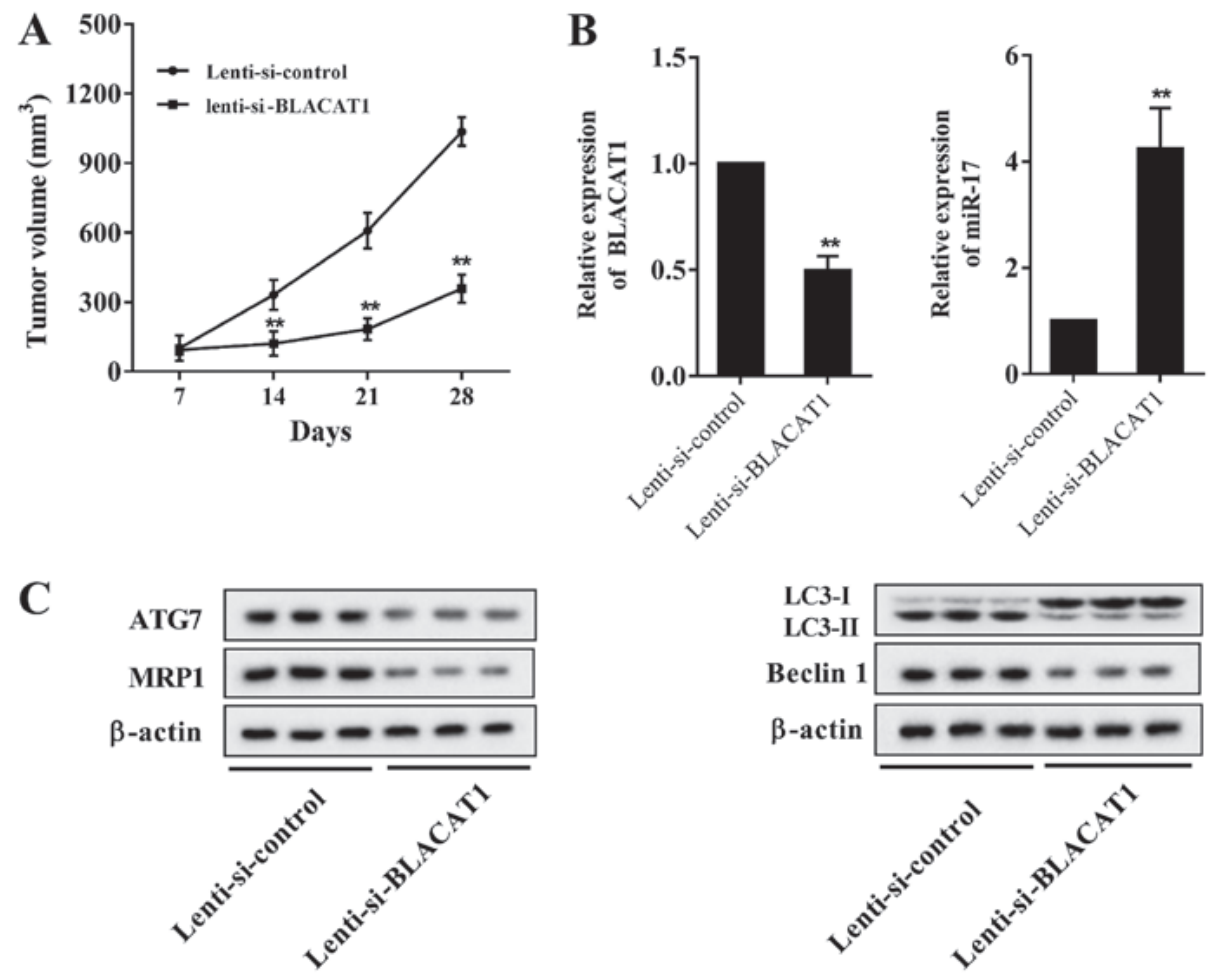

Figure 7. BLACAT1 inhibition ameliorates chemoresistance of non-small cell lung cancer cells in vivo. Lenti-si-control or lenti-si-BLACAT1 was transfected into A549/DDP cells. A total of $1 \times 10^{7}$ A549/DDP cells was subcutaneously injected into male nude mice (BALB/c nu/nu, 5-week-old). Tumor growth was measured using a caliper every 7 days. Mice were sacrificed 28 days after inoculation, and tumor tissues were collected. (A) Tumor volumes in mice of lenti-si-BLACAT1 group were lower compared with those of the lenti-si-control group. (B) BLACAT1 inhibition suppressed BLACAT1 and ATG7 expression, and promoted miR-17 expression. (C) BLACAT1 inhibition suppressed the conversion of LC3-I into LC3-II, and inhibited Beclin 1 and MRP1 expression. ${ }^{* *} \mathrm{P}<0.01$ vs. lenti-si-control. BLACAT1, bladder cancer-associated transcript 1; si, short interfering RNA; DDP, cisplatin; LC3, light chain 3; miR, microRNA; ATG7, autophagy 7; MRP1, multidrug-resistance protein 1.

that IncRNAs regulate the chemoresistance of NSCLC cells $(16,23,27)$. IncRNA BLACAT1, originally called linc-UBC1, was first identified in bladder cancer in 2013 , and was considered as a negative prognostic factor for metastasis to lymph nodes (28). Later, it was identified to be abnormally expressed in colorectal cancer, NSCLC, cervical cancer, etc. $(24,29)$. Previous research has identified that lncRNA BLACAT1 was increased in NSCLC tissues and cell lines, and inhibition of BLACAT1 suppressed NSCLC cell proliferation and migration (24). lncRNA BLACAT1 is also involved in drug resistance of cancer (25); however, the underlying molecular mechanism of BLACAT1 in regulating the chemoresistance of NSCLC cells remains unclear. In the present study, it was identified that IncRNA BLACAT1 was upregulated in DDP-resistant NSCLC cells, which was consistent with results of previous research (24).

A further aim of the present study was to identify the signaling pathway mediated by lncRNA BLACAT1 in regulating the chemoresistance of NSCLC cells. It was identified that miR-17 was downregulated in DDP-resistant NSCLC cells, which was consistent with results of a previous study (20). Furthermore, miR-17 is involved in modulating the cell viability and chemoresistance in a number of types of cancer, such as pancreatic and breast cancer $(30,31)$. According to the bioinformatics software, there were binding sites between BLACAT1 and miR-17. Therefore, IncRNA BLACAT1 may be involved in chemoresistance of NSCLC cells via miR-17. Using RIP and RNA pull-down assays, the interaction between
BLACAT1 and miR-17 was confirmed. Following transfection with pcDNA-BLACAT1 or si-BLACAT1, miR-17 expression was markedly decreased in A549 cells or increased in A549/DDP cells, respectively, which indicated that BLACAT1 negatively regulated miR-17.

Research has focused on the function of autophagy in chemoresistance of cancer cells, but the function of autophagy in the response of cancer cells to chemoresistance is complex and remains unclear $(32,33)$. ATG7, as an autophagy-associated protein, promoted autophagy and enhanced chemoresistance of cancer cells $(14,34)$. On the basis of the negative regulation of miR-17 on ATG7 (21), we hypothesized that lncRNA BLACAT1 may promote autophagy thus to enhance chemoresistance of NSCLC cells via miR-17/ATG7. In the present study, it was identified that the protein level of ATG7 was upregulated in DDP-resistant NSCLC cells, and BLACAT1 positively regulated ATG7. Furthermore, si-BLACAT1 suppressed autophagy-associated protein and multidrug-resistance-associated protein expression in DDP-resistant NSCLC cells, which indicated that inhibition of BLACAT1 could suppress autophagy and chemoresistance of NSCLC cells.

In conclusion, lncRNA BLACAT1 was upregulated in DDP-resistant NSCLC cells, and promoted autophagy and chemoresistance of NSCLC cells. The interaction between BLACAT1 and miR-17 was investigated, and identified the effect of BLACAT1/miR-17/ATG7 pathway in chemoresistance of NSCLC cells, which provided potential targets for the treatment of NSCLC. 


\section{Acknowledgements}

Not applicable.

\section{Funding}

The present study was supported by the Youth Innovation Fund of The First Affiliated Hospital of Zhengzhou University (grant no. YNQN 2017170) and the Science and Technology Key Program of Henan Province (grant no. 162102310196).

\section{Availability of data and materials}

The datasets used and analyzed during the current study are available from the corresponding author on reasonable request.

\section{Authors' contributions}

FXH and LJM designed the study. FXH and HJC drafted the paper. FXH, HJC, FXZ, ZYG and PFS conducted the experiments. QP, YL, XD, YHH and CZ analyzed the data. FXH, HJC and LJM revised the paper.

\section{Ethics approval and consent to participate}

The study was approved by the Ethics Committee of The First Affiliated Hospital of Zhengzhou University.

\section{Patient consent for publication}

Not applicable.

\section{Competing interests}

The authors declare that they have no competing interests.

\section{References}

1. Sapalidis K, Zarogoulidis P, Pavlidis E, Laskou S, Katsaounis A, Koulouris C, Giannakidis D, Mantalovas S, Huang H, Bai C, et al: Aerosol Immunotherapy with or without Cisplatin for metastatic lung cancer non-small cell lung cancer disease: In vivo Study. A more efficient combination. J Cancer 9: 1973-1977, 2018.

2. Bach DH, Kim D, Bae SY, Kim WK, Hong JY, Lee HJ, Rajasekaran N, Kwon S, Fan Y, Luu TT, et al: Targeting Nicotinamide N-Methyltransferase and miR-449a in EGFR-TKI-Resistant Non-Small-Cell Lung Cancer Cells. Mol Ther Nucleic Acids 11: 455-467, 2018.

3. Boya P, Reggiori F and Codogno P: Emerging regulation and functions of autophagy. Nat Cell Biol 15: 713-720, 2013.

4. Kim M, Jung JY, Choi S, Lee H, Morales LD, Koh JT, Kim SH, Choi YD, Choi C, Slaga TJ, et al: GFRA1 promotes cisplatin-induced chemoresistance in osteosarcoma by inducing autophagy. Autophagy 13: 149-168, 2017.

5. Mani J, Vallo S, Rakel S, Antonietti P, Gessler F, Blaheta R, Bartsch G, Michaelis M, Cinatl J, Haferkamp A, et al: Chemoresistance is associated with increased cytoprotective autophagy and diminished apoptosis in bladder cancer cells treated with the BH3 mimetic (-)-Gossypol (AT-101). BMC Cancer 15: 224, 2015.

6. Kim TW, Lee SY, Kim M, Cheon C, Jang BH, Shin YC and Ko SG: DSGOST regulates resistance via activation of autophagy in gastric cancer. Cell Death Dis 9: 649, 2018.

7. Hong SB, Kim BW, Lee KE, Kim SW, Jeon H, Kim J and Song HK: Insights into noncanonical E1 enzyme activation from the structure of autophagic E1 Atg7 with Atg8. Nat Struct Mol Biol 18: 1323-1330, 2011.
8. Noda NN,SatooK, Fujioka Y,KumetaH,Ogura K,Nakatogawa H, Ohsumi Y and Inagaki F: Structural basis of Atg8 activation by a homodimeric E1, Atg7. Mol Cell 44: 462-475, 2011.

9. Yamaguchi M, Satoo K, Suzuki H, Fujioka Y, Ohsumi Y, Inagaki F and Noda NN: Atg7 Activates an Autophagy-Essential Ubiquitin-like Protein Atg8 through Multi-Step Recognition. J Mol Biol 430: 249-257, 2018.

10. Wang G, Chen EN, Liang C, Liang J, Gao LR, Chuai M, Münsterberg A, Bao Y, Cao L and Yang X: Atg7-Mediated Autophagy Is Involved in the Neural Crest Cell Generation in Chick Embryo. Mol Neurobiol 55: 3523-3536, 2018.

11. Gonzalez Y, Aryal B, Chehab L and Rao VA: Atg7- and Keap1-dependent autophagy protects breast cancer cell lines against mitoquinone-induced oxidative stress. Oncotarget 5: 1526-1537, 2014.

12. Hu J, Zhang L, Mei Z, Jiang Y, Yi Y, Liu L, Meng Y, Zhou L, Zeng J, Wu H, et al: Interaction of E3 ubiquitin ligase MARCH7 with long noncoding RNA MALAT1 and autophagy-related protein ATG7 promotes autophagy and invasion in ovarian cancer. Cell Physiol Biochem 47: 654-666, 2018.

13. Mandelbaum J, Rollins N, Shah P, Bowman D, Lee JY, Tayber O, Bernard H, LeRoy P, Li P, Koenig E, et al: Identification of a lung cancer cell line deficient in atg7-dependent autophagy. Autophagy: Jun 19, 2015 (Epub ahead of print).

14. Desai S, Liu Z, Yao J, Patel N, Chen J, Wu Y, Ahn EE, Fodstad O and Tan M: Heat shock factor 1 (HSF1) controls chemoresistance and autophagy through transcriptional regulation of autophagy-related protein 7 (ATG7). J Biol Chem 288: 9165-9176, 2013.

15. Xu N, Zhang J, Shen C, Luo Y, Xia L, Xue F and Xia Q: Cisplatin-induced downregulation of miR-199a-5p increases drug resistance by activating autophagy in HCC cell. Biochem Biophys Res Commun 423: 826-831, 2012.

16. Sun W, Zu Y, Fu X and Deng Y: Knockdown of lncRNA-XIST enhances the chemosensitivity of NSCLC cells via suppression of autophagy. Oncol Rep 38: 3347-3354, 2017.

17. Fabian MR, Sonenberg N and Filipowicz W: Regulation of mRNA translation and stability by microRNAs. Annu Rev Biochem 79: 351-379, 2010.

18. Filipska M, Skrzypski M, Czetyrbok K, Stokowy T, Stasiłojć G, Supernat A, Jassem J, Żaczek AJ and Bigda J: MiR-192 and miR-662 enhance chemoresistance and invasiveness of squamous cell lung carcinoma. Lung Cancer 118: 111-118, 2018.

19. Wei X, Shen X, Ren Y and Hu W: The roles of micrornas in regulating chemotherapy resistance of non-small cell lung cancer. Curr Pharm Des 23: 5983-5988, 2018.

20. Zhao J, Fu W, Liao H, Dai L, Jiang Z, Pan Y, Huang H, Mo Y, Li S, Yang G, et al: The regulatory and predictive functions of miR-17 and miR-92 families on cisplatin resistance of non-small cell lung cancer. BMC Cancer 15: 731, 2015.

21. Comincini S, Allavena G, Palumbo S, Morini M, Durando F, Angeletti F, Pirtoli L and Miracco C: microRNA-17 regulates the expression of ATG7 and modulates the autophagy process, improving the sensitivity to temozolomide and low-dose ionizing radiation treatments in human glioblastoma cells. Cancer Biol Ther 14: 574-586, 2013.

22. Hu B, Zhang H, Wang Z, Zhang F, Wei H and Li L: LncRNA CCAT1/miR-130a-3p axis increases cisplatin resistance in non-small-cell lung cancer cell line by targeting SOX4. Cancer Biol Ther 18: 974-983, 2017.

23. Wang P, Chen D, Ma H and Li Y: LncRNA SNHG12 contributes to multidrug resistance through activating the MAPK/Slug pathway by sponging miR-181a in non-small cell lung cancer. Oncotarget 8: 84086-84101, 2017.

24. Ye JR, Liu L and Zheng F: Long noncoding RNA bladder cancer associated transcript 1 promotes the proliferation, migration, and invasion of nonsmall cell lung cancer through sponging miR-144. DNA Cell Biol 36: 845-852, 2017.

25. Wu X, Zheng Y, Han B and Dong X: Long noncoding RNA BLACAT1 modulates ABCB1 to promote oxaliplatin resistance of gastric cancer via sponging miR-361. Biomed Pharmacother 99: 832-838, 2018.

26. Livak KJ and Schmittgen TD: Analysis of relative gene expression data using real-time quantitative PCR and the 2(-Delta Delta C(T)) Method. Methods 25: 402-408, 2001.

27. Zhang W, Cai X, Yu J, Lu X, Qian Q and Qian W: Exosome-mediated transfer of lncRNA RP11 838N2.4 promotes erlotinib resistance in non-small cell lung cancer. Int J Oncol 53: 527-538, 2018. 
28. He W, Cai Q, Sun F, Zhong G, Wang P, Liu H, Luo J, Yu H, Huang $\mathrm{J}$ and Lin T: linc-UBC1 physically associates with polycomb repressive complex 2 (PRC2) and acts as a negative prognostic factor for lymph node metastasis and survival in bladder cancer. Biochim Biophys Acta 1832: 1528-1537, 2013.

29. Su J, Zhang E, Han L, Yin D, Liu Z, He X, Zhang Y, Lin F, Lin Q, Mao P, et al: Long noncoding RNA BLACAT1 indicates a poor prognosis of colorectal cancer and affects cell proliferation by epigenetically silencing of p15. Cell Death Dis 8: e2665, 2017.

30. Gu J, Wang D, Zhang J, Zhu Y, Li Y, Chen H, Shi M, Wang X, Shen B, Deng X, et al: GFR $\alpha 2$ prompts cell growth and chemoresistance through down-regulating tumor suppressor gene PTEN via Mir-17-5p in pancreatic cancer. Cancer Lett 380: 434-441, 2016.

31. Ao X, Nie P, Wu B, Xu W, Zhang T, Wang S, Chang H and Zou Z: Decreased expression of microRNA-17 and microRNA-20b promotes breast cancer resistance to taxol therapy by upregulation of NCOA3. Cell Death Dis 7: e2463, 2016.
32. Notte A, Leclere L and Michiels C: Autophagy as a mediator of chemotherapy-induced cell death in cancer. Biochem Pharmacol 82: 427-434, 2011

33. Wu WK, Coffelt SB, Cho CH, Wang XJ, Lee CW, Chan FK, Yu J and Sung JJ: The autophagic paradox in cancer therapy. Oncogene 31: 939-953, 2012.

34. Piya S, Kornblau SM, Ruvolo VR, Mu H, Ruvolo PP, McQueen T, Davis RE, Hail N Jr, Kantarjian H, Andreeff M, et al: Atg7 suppression enhances chemotherapeutic agent sensitivity and overcomes stroma-mediated chemoresistance in acute myeloid leukemia. Blood 128: 1260-1269, 2016. 